\title{
Individual capacity differences predict working memory performance and prefrontal activity following dopamine receptor stimulation
}

\author{
SASHA E. B. GIBBS and MARK D'ESPOSITO \\ University of California, Berkeley, Califormia
}

\begin{abstract}
Dopamine receptors are abundant in the prefrontal cortex (PFC), a critical region involved in working memory. This pharmacological fMRI study tested the relationships between dopamine, PFC function, and individual differences in working memory capacity. Subjects performed a verbal delayed-recognition task after taking either the dopamine receptor agonist bromocriptine or a placebo. Behavioral effects of bromocriptine treatment depended on subjects' working memory spans, with the greatest behavioral benefit for lower span subjects. After bromocriptine, PFC activity was positively correlated with a measure of cognitive efficiency (RT slope) during the probe period of the task. Less efficient subjects with slower memory retrieval rates had greater PFC activity, whereas more efficient subjects had less activity. After placebo, these measures were uncorrelated. These results support the role of dopamine in verbal working memory and suggest that dopamine may modulate the efficiency of retrieval of items from the contents of working memory. Individual differences in PFC dopamine receptor concentration may thus underlie the behavioral effects of dopamine stimulation on working memory function.
\end{abstract}

Working memory refers to those processes that support the short-term maintenance or manipulation of relevant information when it is no longer present to guide behavior. Monkey physiology and human functional neuroimaging studies support the critical involvement of the prefrontal cortex (PFC) in working memory (Curtis \& D'Esposito, 2003; Fuster, 1997). The PFC is the main target of extensive dopamine projections from the midbrain. In addition, the nigrostriatal dopaminergic pathway innervates the basal ganglia, which also have direct and extensive connections with PFC (Cooper, Bloom, \& Roth, 1991). An early study demonstrated that depleting PFC dopamine, but not norepinephrine or serotonin, caused performance deficits equivalent to PFC lesions in monkeys performing a delayed-response task (Brozoski, Brown, Rosvold, \& Goldman, 1979). Later animal work demonstrated that endogenous baseline dopamine levels and evoked release of dopamine influence performance on delayed-response tasks. For example, the amount of dopamine released in the PFC is positively correlated with accurate memory performance (Floresco \& Phillips, 2001; Phillips, Ahn, \& Floresco, 2004). Also, delayedresponse tasks elicit increases in extracellular PFC dopamine that are not seen when performance is guided by

This work was supported by a training fellowship to S.E.B.G. from the Wheeler Center for the Neurobiology of Addiction and by NIH Grants DA11754 and MH63901 to M.D. The authors gratefully acknowledge valuable comments and suggestions from Roshan Cools, Adam Gazzaley, and Eric Schumacher and imaging support from Ben Inglis. Correspondence relating to this article may be sent to S. E. B. Gibbs, Department of Psychology, Stanford University, 450 Serra Mall, Building 420, Stanford, CA 94305-2130 (e-mail: sebgibbs@psych.stanford.edu). visual cues alone (Watanabe, Kodama, \& Hikosaka, 1997). Application of dopamine to the PFC increases task-related activity, and it does so by increasing the signal-to-noise ratio (Durstewitz \& Seamans, 2002; Sawaguchi, Matsumura, \& Kubota, 1990b).

There is also evidence suggesting that D1 and D2 receptor family activity may affect different components of working memory. Since there are currently no D1-selective agonists approved for human use, most substantiating evidence comes from neurophysiological studies of primates performing delayed-recognition tasks. Delay-related processing may be most sensitive to D1 receptor activation, because D1 agonists increase and D1 antagonists reduce delay period activity in the primate PFC (Sawaguchi, 2001; Sawaguchi, Matsumura, \& Kubota, 1988). In contrast, effects of selective D2 receptor agonists and antagonists suggest that D2 receptor activation is most critical for response preparation processes (Wang, Vijayraghavan, \& Goldman-Rakic, 2004). Thus, dopamine appears to play a critical role in working memory function, and activation of different receptor families may selectively influence component processes of working memory (Cohen, Braver, \& Brown, 2002; Cools \& Robbins, 2004).

Human studies have also provided support for a relationship between dopamine and working memory. For example, young subjects' performance on a spatial delayedresponse task was improved by low doses of bromocriptine, a D2 dopamine receptor agonist (Luciana \& Collins, 1997; Luciana, Depue, Arbisi, \& Leon, 1992), and pergolide, a mixed D1-D2 receptor agonist (Müller, von Cramon, \& Pollmann, 1998). Bromocriptine has also been shown to improve working memory in patients with 
PFC lesions (McDowell, Whyte, \& D’Esposito, 1998). Further support for a relationship between dopamine and working memory comes from the finding that sulpiride, a nonspecific dopamine antagonist, causes behavioral deficits on tasks of spatial working memory (Mehta, Sahakian, McKenna, \& Robbins, 1999).

Many lines of research have suggested an inverted-U shaped relationship between dopamine levels and PFC function. For example, application of dopaminergic drugs to PFC neurons can enhance delay period activity at one concentration but inhibit firing at other dosages (Williams \& Goldman-Rakic, 1995). Also, larger increases in PFC dopamine from noise stress (Arnsten \& Goldman-Rakic, 1998) or with direct application of dopaminergic agents (Zahrt, Taylor, Mathew, \& Arnsten, 1997) cause behavioral impairment. Thus, there likely is an optimal level of dopaminergic stimulation that is necessary for maximizing the signal-to-noise ratio in a population of PFC neurons that support mnemonic processes. It seems likely that the effects of PFC dopamine receptor stimulation on working memory will also depend on baseline dopamine receptor concentration, a property that varies among individuals.

Evidence suggests that individual differences in baseline dopamine receptor concentration may exist and may account for the behavioral effect of dopamine agonists on working memory. For example, poorly performing young rats (Granon et al., 2000) and monkeys with working memory impairments from age-related dopamine loss (Castner \& Goldman-Rakic, 2004) or reserpine depletion (Arnsten, Cai, Steere, \& Goldman-Rakic, 1995) derive the greatest behavioral benefit from agonist treatment. This finding suggests suboptimal baseline dopamine receptor occupation in these poorly performing animals. Similarly, administering dopamine agonists to human subjects can improve or impair working memory performance depending on the subject's baseline working memory capacity, which may reflect underlying baseline PFC dopamine receptor concentration. Subjects with low working memory capacity, as measured by a span task, show improvements in PFC-dependent tasks with low doses of the D2 dopamine agonist bromocriptine (Kimberg, D'Esposito, \& Farah, 1997) or the nonselective dopamine agonist methylphenidate (Mehta et al., 2000), whereas their high-span counterparts are either impaired or show no change in performance. Genotypic variations that are thought to affect PFC dopamine concentration support the link between working memory performance, PFC dopamine availability, and response to dopaminergic drugs. For example, subjects with genetic variation associated with lower PFC dopamine availability have poorer working memory performance at baseline but improve when given the nonspecific agonist amphetamine, whereas those with genetic variation associated with higher PFC dopamine availability have better performance at baseline and are impaired on amphetamine (Goldberg et al., 2003; Mattay et al., 2003)

The present study aims to investigate the relationship between dopamine, PFC function, and individual differ- ences in baseline working memory performance. In this study, brain dopamine levels were manipulated by administering the D2 dopamine receptor agonist bromocriptine while measuring PFC blood oxygenation level dependent (BOLD) signal during functional MRI (fMRI). We assumed that baseline brain dopamine concentration is reflected by baseline working memory capacity, as measured with a span task that has previously been shown to predict the effect of bromocriptine on working memory performance (Kimberg et al., 1997). In the present study, we also used a working memory task that has been shown to produce a correlation between PFC activity and individual differences in working memory performance. In a verbal delayed-recognition task in healthy young unmedicated subjects, a direct relationship was observed between PFC activity and memory retrieval rate (Rypma \& D'Esposito, 1999). Memory retrieval rate was indexed by calculating the reaction time (RT) slope. This measure quantifies the increase in RT required across different loads of information held in working memory (Sternberg, 1966) and reflects those decision processes occurring during the probe, including memory scanning and item selection. Fast, efficient subjects (i.e., lower RT slopes) had less PFC fMRI BOLD signal during retrieval than slower, less efficient subjects (i.e., higher RT slopes) (Rypma \& D’Esposito, 1999). Importantly, an index of motor speed (i.e., the intercept of the RT slope, which estimates the RT with no memory load) did not correlate with PFC activity. It was proposed that the neural correlates of processing efficiency may be reflected as less activity within relevant neural circuits. Thus, we predict that bromocriptine will directly influence this brainbehavior relationship.

Insight regarding how dopaminergic stimulation may influence this verbal recognition task derives from a behavioral study of Parkinson's disease (PD) patients who were tested on and off their dopaminergic replacement medications (Poewe, Berger, Benke, \& Schelosky, 1991). Patients showed facilitated motor speed (decreased RT intercept) and impaired cognitive efficiency (increased RT slope) when they were on their medication in comparison with when they were off their medication. The decreases in RT intercept parallel the expected relief from motor symptoms, but the increased RT slope suggests that cognitive systems were overdosed by the medication, leading to inefficient memory retrieval. Poewe and colleagues' results also support the idea that an optimal dosage of dopamine is necessary for optimal PFC function. In the present study, we predicted that normal healthy subjects with high working memory capacity would also be "overdosed" by bromocriptine, leading to poorer behavioral performance, whereas those with lower working memory performance might improve. Pharmacological manipulation during scanning allows us to directly investigate the neural correlates of these effects. Moreover, we predict that the effects of bromocriptine on the relationship between PFC activity and working memory performance will be influenced by individual 
differences in baseline working memory capacity. By using an event-related design, we were also able to correlate the drug effects with specific stages of processing during our task. Neither of these aims could be achieved in the behavioral study of PD patients.

\section{METHOD}

\section{Subjects}

Thirteen young healthy subjects ( 9 females; ages of all subjects, 21-30) gave written informed consent according to University of California guidelines. The subjects were screened for use of prescription medication, history of neurological or psychiatric disorders, blood pressure abnormality, and any other conditions that would preclude completing the study (e.g., metallic implants, difficulty with manual responses, or low visual acuity). In addition, the subjects were screened for current mood disturbances with the Beck depression inventory and mood assessment scale.

\section{Cognitive Tasks}

The subjects were tested after administration of $1.25 \mathrm{mg}$ bromocriptine or a lactose placebo in a double-blind design. They were scanned approximately $120 \mathrm{~min}$ following administration of the drug or placebo, and the order of sessions was counterbalanced across subjects. fMRI scans were acquired while the subjects performed four types of working memory trials. Two or six blockcapital letters were presented for $4 \mathrm{sec}$, followed by an unfilled 12 -sec delay period. At the end of the delay period, a single lowercase probe letter appeared for $2 \mathrm{sec}$. The probe letter was presented either superimposed upon or masked by a background of visual noise. The subjects indicated whether the probe letter was a part of their memory set with a manual buttonpress. The response period was followed by a jittered intertrial interval lasting between 8 and $12 \mathrm{sec}$. Four trials of each of the four types were presented per 7-min run. There were six runs, for a total of 96 trials per scanning session.

We administered different memory load levels in order to calculate the RT slope and RT intercept for each subject. We chose two levels based on prior reports that RT increases linearly with set size (Sternberg, 1966, 1967a, 1969a) and that the RT intercept estimates motor speed under conditions of no memory load (Sternberg, 1967b, 1969b). We also included probe stimuli that were intact or degraded with visual noise. Manipulating probe stimulus discriminability has been shown to selectively slow processes related to item identification without affecting memory scanning rates (Hardzinski \& Pachella, 1980; Irwin \& Pachella, 1985; Pachella \& Fisher, 1969; Sternberg, $1967 \mathrm{~b}$ ); thus, the degradation factor may selectively manipulate RT intercept without changing RT slope.

The subjects were also given control tasks assessing motor speed and vigilance before and after the drug and placebo scanning sessions. Motor speed was measured with a box completion task. The subjects were presented with 10 rows of 10 line drawings of squares, each with one side missing. They were asked to draw in the missing side of each square as quickly and accurately as possible. We then administered a numeral cancellation task to measure vigilance (the digit vigilance test; Lewis \& Kupke, 1977), in which the subjects crossed out target digits from a field of 28 rows of 35 digits as quickly and accurately as possible. Finally, in a separate session, the subjects performed the listening version of the Daneman and Carpenter reading span task (Daneman \& Carpenter, 1980).

\section{MRI Data Acquisition}

Functional and structural images were acquired with a Varian INOVA 4.0T scanner (Palo Alto, CA; www.varianinc.com) and a TEM send-and-receive RF head coil (MR Instruments, Minneapolis, $\mathrm{MN}$; www.mrinstruments.com). Head movement was restricted using a foam cushion adjusted for each subject. The stimuli were backprojected onto a screen at the subject's waist and viewed by means of a mirror mounted inside the head coil.

Functional images were acquired using a 2-shot gradient echo EPI sequence, in eighteen 5.0 -mm thick axial slices with a $0.5-\mathrm{mm}$ interslice gap and a TR of 2,000 msec. Each slice was acquired with a $22.4-\mathrm{cm}^{2}$ field of view with a $64 \times 64$ matrix size, resulting in an in-plane resolution of $3.5 \times 3.5 \mathrm{~mm}$. This slice prescription allowed for near whole-brain coverage. Data were acquired during six runs lasting $7 \mathrm{~min}$ each. The first 10 images from each run were deleted to approach steady-state tissue magnetization. High-resolution inplane $\mathrm{T} 1$-weighted images were acquired using a gradient echo multislice sequence for anatomical localization. In addition, highresolution MP-Flash 3-D T1-weighted scans were acquired for normalization to the Montreal Neurological Institute (MNI) reference brain.

\section{MRI Data Analysis}

Functional images acquired from the scanner were reconstructed offline from k-space. Image volumes were corrected for slice timing skew using temporal sync-interpolation and corrected for movement using rigid-body transformation parameters. Image preprocessing and statistical analyses were performed using SPM2 (Wellcome Department of Imaging Neuroscience; www.fil.ion.ucl.ac.uk/spm). Images were resampled to $2 \times 2 \times 2 \mathrm{~mm}$ and then smoothed with an 8-mm FWHM Gaussian kernel. A high-pass filter was used to remove frequencies below $0.01 \mathrm{~Hz}$ from the data.

Structural T1-weighted images were normalized to the MNI reference brain. Transformations calculated from normalizing each subject's structural images were applied to the functional images collected in each run. Data were analyzed using the general linear model (Worsley \& Friston, 1995). For each subject, the BOLD signals during the cue, delay, and probe periods in each trial type were modeled as impulses of neural activity convolved with the SPM canonical hemodynamic response function. We used a covariate beginning at the onset of the cue period (first TR, $0 \mathrm{sec}$ ) to model early encoding processes. A second covariate at the third TR $(4 \mathrm{sec})$ modeled late encoding activity. Since encoding processes may continue into the delay period, this late encoding period is modeled to reduce noise in the estimate of the baseline but was not included in the mapwise analysis (Zarahn, Aguirre, \& D'Esposito, 1999). The early and late phases of the delay period were modeled with covariates at the fifth and seventh TRs, respectively (8 and $12 \mathrm{sec}$ ). The delay period effects reported here represent the sum of the two delay period regressors. The retrieval period was modeled with a covariate at the onset of the probe (ninth TR, $16 \mathrm{sec}$ ). These covariates were then entered into the general linear model (GLM). Maps of parameter estimates were computed from the GLM to assess the magnitude of activation during each trial period. Stereotactic coordinates of peak activations were reported with respect to the MNI coordinate system.

Group random-effects analyses were performed for each contrast of interest to test whether the mean parameter estimate magnitude was significantly different from zero. Areas of significant activation in the individual-session mapwise analyses were determined by identifying regions in which peak activation exceeded a threshold of $t=3.93$ ( $p<.001$, uncorrected). PFC BOLD signal is often more variable across individuals during the delay period than at cue or probe presentation (Druzgal \& D'Esposito, 2003), and this factor affects sensitivity in group analyses. This may be related to subtle differences in the shape of the hemodynamic response function in different brain regions or to the sensitivity of various methods of modeling the duration of neural activity. To compare task-related activity during all task periods, we selected a delay period $\mathrm{PFC}$ region using a threshold of $t=3.09$ ( $p<.005$, uncorrected). We then performed a conjunction analysis to isolate task-related regions common to both the drug and placebo sessions within each distinct 
stage of the task. These regions were tested for drug effects, because this contrast is orthogonal to the main effect of task contrast.

\section{RESULTS}

\section{Behavioral Data}

Group effects. Using a within-subjects analysis of variance (ANOVA), we examined the effect of session (bromocriptine vs. placebo) and administration time (prescan vs. postscan) on performance for the control tasks of motor speed and vigilance (box completion and numeral cancellation tasks). There was no effect of session [box completion, $F(1,12)=0.43, p=.52$; cancellation, $F(1,12)=0.28, p=.61]$ or administration time [box completion, $F(1,12)=2.26, p=.16$; cancellation, $F(1,12)=0.21, p=.66]$ and no interaction between the two factors [box completion, $F(1,12)=0.02, p=.89$; cancellation, $F(1,12)=0.42, p=.53]$.

We also performed a within-subjects ANOVA to test the effects of load, perceptual degradation, and bromocriptine on RT and accuracy for the working memory task (Table 1). There was a main effect of load on reaction time $[F(1,12)=48.77, p<.001]$ in which subjects were slower at the higher memory load in both sessions (bromocriptine, $t=8.16, p<.001$; placebo, $t=5.41$, $p<.001)$. Similarly, there was a significant effect of load on accuracy $[F(1,12)=8.85, p=.012]$ : Subjects were less accurate at the higher load in both the bromocriptine $(t=2.66, p=.02)$ and placebo sessions $(t=$ $2.52, p=.03)$. Since the perceptual degradation manipulation did not reliably affect $\mathrm{RT}[F(1,12)=3.32, p=$ $.09]$ or accuracy $[F(1,12)=0.08, p=.79]$, we collapsed our subsequent analyses across perceptual conditions.

There was no main effect of bromocriptine treatment on reaction time $[F(1,12)=1.20, p=.30]$ or accuracy $[F(1,12)=0.05, p=.82]$ and no interactions among any of the factors (all $p \mathrm{~s}>.2$ ).

Individual differences. Working memory capacity was estimated in each subject using a listening span task (Daneman \& Carpenter, 1980). The range of spans in our group of subjects was from 2.5 to 6.5 items, with a mean score of $4.07 \pm S D$ 1.35. As a reference point, in the original report of this task by Daneman and Carpenter, the observed range of spans was 2 to 4.5 , with a mean of $2.95 \pm 0.72$. In the behavioral analyses reported below, we compared those individuals with a high span (4 or more items, $n=8)$ with those with lower span $(<4$ items, $n=5$ ). This method is based on previous studies (Kimberg et al., 1997; Mehta et al., 2000) and was intended to be independent from the behavioral and imaging data collected in this study.

Our first analyses concerned differences in performance between low-span and high-span subjects during the placebo condition. As is shown in Table 1, accuracy was very high and near ceiling at low load for both lowand high-span subjects and slightly but not significantly higher for high-span subjects at high loads. We predicted that behavioral differences between the groups should be most apparent in RT slope (i.e., the difference in RTs between high and low loads), since RT slope is sensitive to differences in memory retrieval efficiency. In accord with our prediction, RT slope was quite different between groups (46 msec/item in low-span subjects vs. $25 \mathrm{msec} /$ item in high-span subjects).

Next, we investigated interactions between drug treatment and memory span. With RT, there was an interaction between the effects of bromocriptine and working memory capacity $[F(1,11)=4.69, p=.05]$ and another between load, bromocriptine, and working memory capacity $[F(1,11)=5.44, p=.04]$. Planned comparisons revealed that this pattern was due to the lack of effect of bromocriptine on high-span subjects' performance at either load $(p>.4)$ and a trend to faster RTs for low-span subjects at high load $(t=-2.03, p=.11)$. With accuracy, there were also interactions between the effects of bromocriptine and working memory $\operatorname{span}[F(1,11)=$ $6.76, p=.03]$ and between load, bromocriptine treatment, and working memory span $[F(1,11)=5.48, p=$ .04]. Planned comparisons revealed that low-span subjects became more accurate on bromocriptine when performing the high-load trials ( $83 \%$ on placebo vs. $90 \%$ on bromocriptine; $t=6.67, p=.003)$. In contrast, highspan subjects on bromocriptine showed a trend to reduced accuracy on high-load trials ( $85 \%$ on placebo vs. $78 \%$ on bromocriptine; $t=-2.13, p=.07)$. There were no reliable differences in the performance of low-load trials (both $p \mathrm{~s}>.11$ ).

In addition to their accuracy improvements, low-span subjects had significant reductions in RT slope on bromocriptine (placebo, $46.2 \mathrm{msec} /$ item; bromocriptine, $32.0 \mathrm{msec} /$ item), indicating faster processing speed $(t=-2.86, p=$ $.05)$. There was no difference in RT slope between the

Table 1

Comparison of Behavioral Data for High- and Low-Span Subjects on Bromocriptine and Placebo (Mean $\pm S D$ )

\begin{tabular}{|c|c|c|c|c|c|c|c|c|c|}
\hline \multirow[b]{2}{*}{ Subjects } & \multicolumn{2}{|c|}{ RT Slope (msec) } & \multicolumn{2}{|c|}{ Intercept (msec) } & \multirow[b]{2}{*}{ Memory Load } & \multicolumn{2}{|c|}{ Reaction Time (msec) } & \multicolumn{2}{|c|}{ Accuracy $(\%)$} \\
\hline & romocriptine & Placebo & Bromocriptine & Placebo & & Bromocriptine & Placebo & Bromocriptine & Placebo \\
\hline All & $30 \pm 13$ & $33 \pm 22$ & $1,005 \pm 22$ & $1,019 \pm 237$ & & $\begin{array}{l}1,127 \pm 201 \\
1,248 \pm 198\end{array}$ & $1,285 \pm 142$ & $\begin{array}{r}89 \pm 13 \\
83.3 \pm 19\end{array}$ & $\begin{array}{l}89 \pm 11 \\
84 \pm 13\end{array}$ \\
\hline High-span & $29 \pm 7$ & $25 \pm 18$ & $1,065 \pm 218$ & $1,080 \pm 265$ & $\begin{array}{l}\text { Two letters } \\
\text { Six letters }\end{array}$ & $\begin{array}{l}1,183 \pm 213 \\
1,300 \pm 211\end{array}$ & $\begin{array}{l}1,180 \pm 213 \\
1,280 \pm 176\end{array}$ & $\begin{array}{l}86 \pm 16 \\
78 \pm 23\end{array}$ & $\begin{array}{l}88 \pm 13 \\
85 \pm 17\end{array}$ \\
\hline Low-span & $32 \pm 21$ & $46 \pm 24$ & $909 \pm 216$ & $923 \pm 262$ & $\begin{array}{l}\text { Two letters } \\
\text { Six letters }\end{array}$ & $\begin{array}{l}1,038 \pm 162 \\
1,166 \pm 141\end{array}$ & $\begin{array}{l}1,108 \pm 85 \\
1,292 \pm 83\end{array}$ & $\begin{array}{l}93 \pm 2 \\
90 \pm 5\end{array}$ & $\begin{array}{l}90 \pm 5 \\
83 \pm 6\end{array}$ \\
\hline
\end{tabular}


drug and placebo sessions for high-span subjects $(t=0.79$, $p=.45$ ), although the RT slope was numerically higher for the drug session (placebo, $26.1 \mathrm{msec} /$ item; bromocriptine, $29.1 \mathrm{msec} /$ item). There were no differences in RT intercept for the two span groups (both $p \mathrm{~s}>.62$ ).

\section{Imaging Data}

Task-related PFC activity. All analyses that follow examine $\mathrm{PFC}$ regions that were active during each task period (i.e., cue, delay, and probe). PFC regions were first identified in the drug and placebo sessions separately, and a conjunction analysis then identified regions common to both sessions. In agreement with many previously published event-related fMRI studies using delayedrecognition paradigms, this analysis revealed PFC activity during all three task periods (Figure 1, Table 2). During the cue period, activity was found in left middle $(-38,32,22 ; 2$ voxels) and inferior frontal gyrus $(-36$, 26,$16 ; 11$ voxels) and right inferior frontal gyrus $(38$, 10,$20 ; 191$ voxels). During the delay period, activity was found in left inferior frontal gyrus $(-52,40,14$; 5 voxels). During the probe period, activity was observed in left middle frontal gyrus $(-32,4,66 ;-40,46,30 ;-44$, 32,$30 ; 114$ voxels), left inferior frontal gyrus $(-38,32$, $28 ;-40,12,28 ; 107$ voxels), right middle frontal gyrus $(28,36,26 ; 314$ voxels), and right inferior frontal gyrus $(42,12,24 ; 262$ voxels). For reference, results of the mapwise analysis showing all regions active in both sessions are presented in Table 2.

Brain-behavior relationships. We chose a task that has been shown in prior studies to differentially activate the PFC for subjects with fast and slow memory-scanning rates. Effects of bromocriptine treatment differ for sub- jects with high and low working memory capacity. Thus, individual differences were tested in two ways: by examining the effects of bromocriptine on the relationship between memory retrieval rate and PFC activity and by investigating whether these effects on memory scanning differed for subjects with high and low working memory capacity.

To test our initial hypothesis that dopamine receptor stimulation affects the relationship between memory retrieval rate and PFC activity, we examined the correlations in each subject between the magnitude of their PFC activity during each of the task periods and the two component measures of reaction time: RT slope, an index of cognitive processing speed, and RT intercept, an index of motor processing speed.

In the bromocriptine session, during the probe period PFC activity was correlated with processing speed, so that slower subjects (i.e., higher RT slope) had increased and faster subjects (i.e., lower RT slope) had decreased right middle frontal gyrus activity $(r=.56, p=.05$; see Figure 2). This pattern was also observed in right inferior frontal gyrus $(r=.52, p=.07)$ and left middle frontal gyrus $(r=.50, p=.08$; see Figure 2$)$. In contrast, during the placebo session there was no relationship between processing speed and PFC activity (right middle frontal gyrus, $r=-.17, p=.59 ; p>.52$ for all other PFC regions; see Figure 2). PFC activity was not related to RT intercept for any of the regions (all $p \mathrm{~s}>.33$ ). Furthermore, there was no relationship between PFC activity and RT slope or intercept during the cue and delay periods (all $p \mathrm{~s}>.39$ ).

To illustrate how drug-induced changes in PFC activity with accompanying changes in RT slope are related

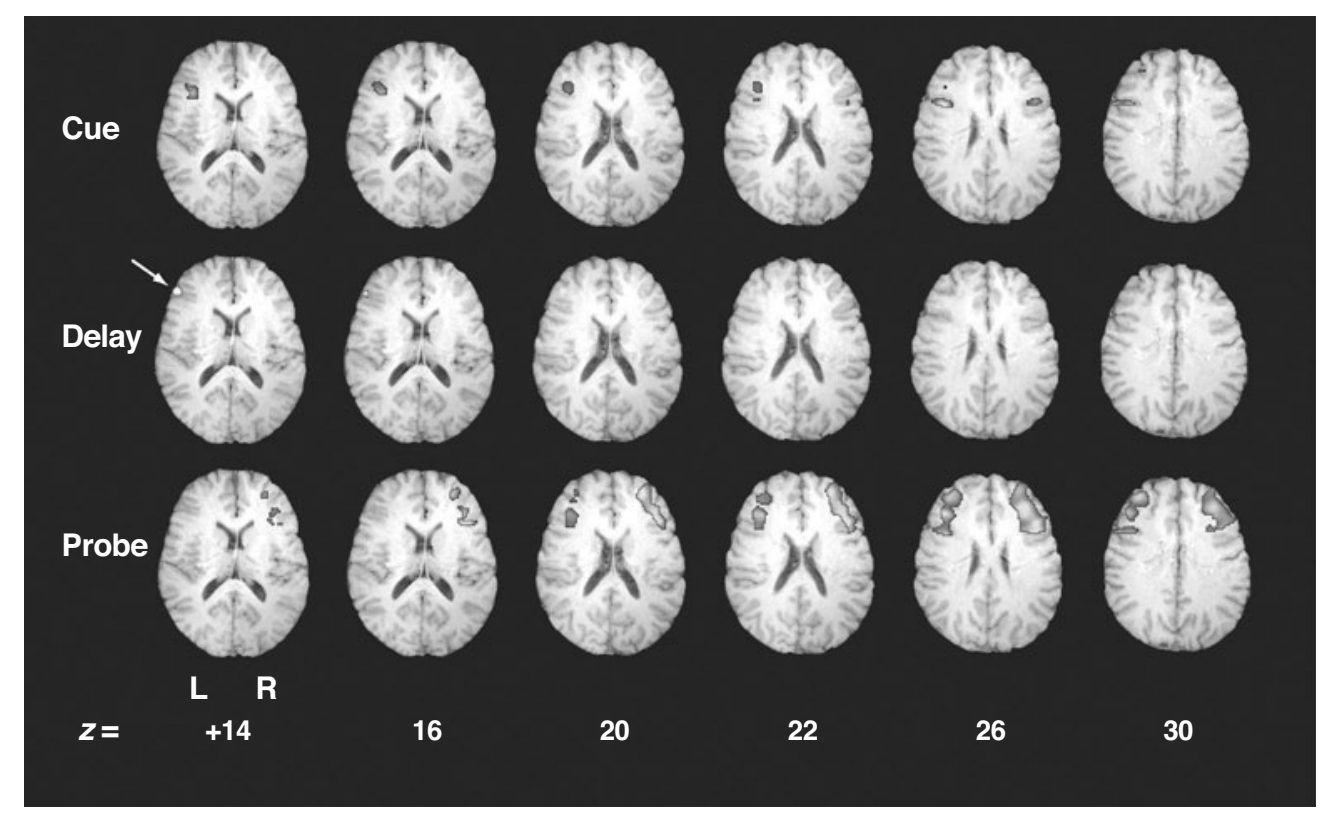

Figure 1. Regions of peak PFC activity in both bromocriptine and placebo sessions during each of the task periods, displayed at $\boldsymbol{t}=\mathbf{3 . 0 9}$ for cue and probe periods and $\boldsymbol{t}=\mathbf{2 . 7 5}$ for the delay period. 
Table 2

\begin{tabular}{lcr}
\multicolumn{2}{c}{$\begin{array}{c}\text { Regions of Interest Showing Increased Activation in Both Bromocriptine and } \\
\text { Placebo Sessions for the Cue, Delay, and Probe Task Periods }\end{array}$} \\
\hline \multicolumn{1}{c}{ Region } & Coordinates $(x, y, z)$ & Peak $t$ \\
\hline Cue Period & & \\
Left middle frontal gyrus & $-38,32,22$ & 3.98 \\
Left inferior frontal gyrus & $-36,26,16$ & 5.21 \\
Left premotor cortex & $-52,0,44$ & 13.50 \\
Left inferior parietal cortex & $-44,-36,36$ & 4.44 \\
Left inferior parietal cortex & $-30,-52,46$ & 7.91 \\
Left middle occipital gyrus & $-38,-80,12$ & 11.62 \\
Supplementary motor area (SMA) & $0,6,60$ & 19.42 \\
Right inferior frontal gyrus & $38,10,20$ & 6.80 \\
Right premotor cortex & $48,0,42$ & 9.81 \\
Bilateral hippocampus/parahippocampal gyrus & $20,-30,-6$ & 18.69 \\
Posterior cingulate gyrus & $4,-30,26$ & 5.43 \\
Right fusiform gyrus & $38,-52,-14$ & 9.19 \\
Right angular gyrus/superior occipital gyrus & $26,-56,44$ & 12.61 \\
Right lingual gyrus/inferior occipital gyrus & $22,-92,-10$ & 11.13 \\
Delay Period & & \\
Left inferior frontal gyrus & $-52,40,14$ & 3.37 \\
Left premotor cortex & $-56,-2,46$ & 11.64 \\
SMA & $-6,2,58$ & 5.86 \\
Brainstem & $0,-18,-16$ & 4.32 \\
Right premotor cortex & $34,-24,62$ & 6.55 \\
Probe Period & & \\
Left inferior frontal gyrus & $-38,32,28$ & 6.18 \\
Left middle frontal gyrus & $-32,4,66$ & 6.88 \\
Left middle temporal gyrus & $-54,-44,8$ & 4.06 \\
Left middle temporal gyrus & $-42,-56,-2$ & 10.37 \\
Left lingual gyrus & $-4,-56,-4$ & 6.34 \\
SMA & $-4,10,50$ & 17.11 \\
Middle cingulate gyrus & $-10,-24,36$ & 3.95 \\
Thalamus/globus pallidus & $16,-4,0$ & 12.58 \\
Right middle frontal gyrus & $28,36,26$ & 5.57 \\
Right inferior frontal gyrus & $42,12,24$ & 6.28 \\
Right premotor cortex & $30,-10,48$ & 12.47 \\
Posterior cingulate gyrus & $4,-34,26$ & 9.01 \\
Right precuneus & $10,-42,48$ & 4.22 \\
Right lingual gyrus & $20,-54,-2$ & 7.81 \\
Right lingual gyrus & $18,-58,-2$ & 9.93 \\
Right fusiform gyrus & $26,-64,-16$ & 8.36 \\
Right middle occipital gyrus & $40,-74,8$ & 5.77 \\
\hline & & \\
& &
\end{tabular}

to working memory span, we plotted the drug effect on PFC BOLD signal against the drug effect on RT slope (Figure 3). In the right middle frontal gyrus, which showed the most significant correlations between RT slope and BOLD signal, $8 / 13$ subjects exhibited drug effects in the predicted direction based on their baseline working memory capacity. That is, high-span subjects had drug-induced increases in PFC activity and slowing of their retrieval rates (6/8 subjects, upper right quadrant of graph), whereas low-span subjects had drug-induced decreases in PFC activity and/or speeding of their retrieval rates $(2 / 5$ subjects; lower left quadrant of graph). Four of the 5 remaining subjects showed drug effects in the predicted direction for either PFC activity or RT slope, but not for both measures.

\section{Global Effects of Bromocriptine}

To address the concern of global vascular effects of bromocriptine, we tested the main effect of bromocriptine collapsed across all task periods and all trial types.
Two regions showed increased global activity on bromocriptine relative to placebo: the right middle temporal gyrus $(58,-72,10)$ and the right angular gyrus $(46,-64$, $50)$. One region in the left middle occipital gyrus $(-20$, $-102,4)$ showed decreased global activity on bromocriptine. No effects were seen in any of the PFC regions, even at a lower threshold $(t=2.75, p<.01$, uncorrected $)$.

\section{DISCUSSION}

This study tested the relationships between dopamine, PFC function, and individual differences in baseline working memory performance. To achieve this aim, we used event-related fMRI to isolate working memory subprocesses associated with the cue, delay, and probe periods of a verbal delayed-recognition task and examined the effect of the D2 dopamine agonist bromocriptine on these processes. We found that the behavioral response to bromocriptine treatment depended on subjects' baseline working memory capacity as measured by a listen- 

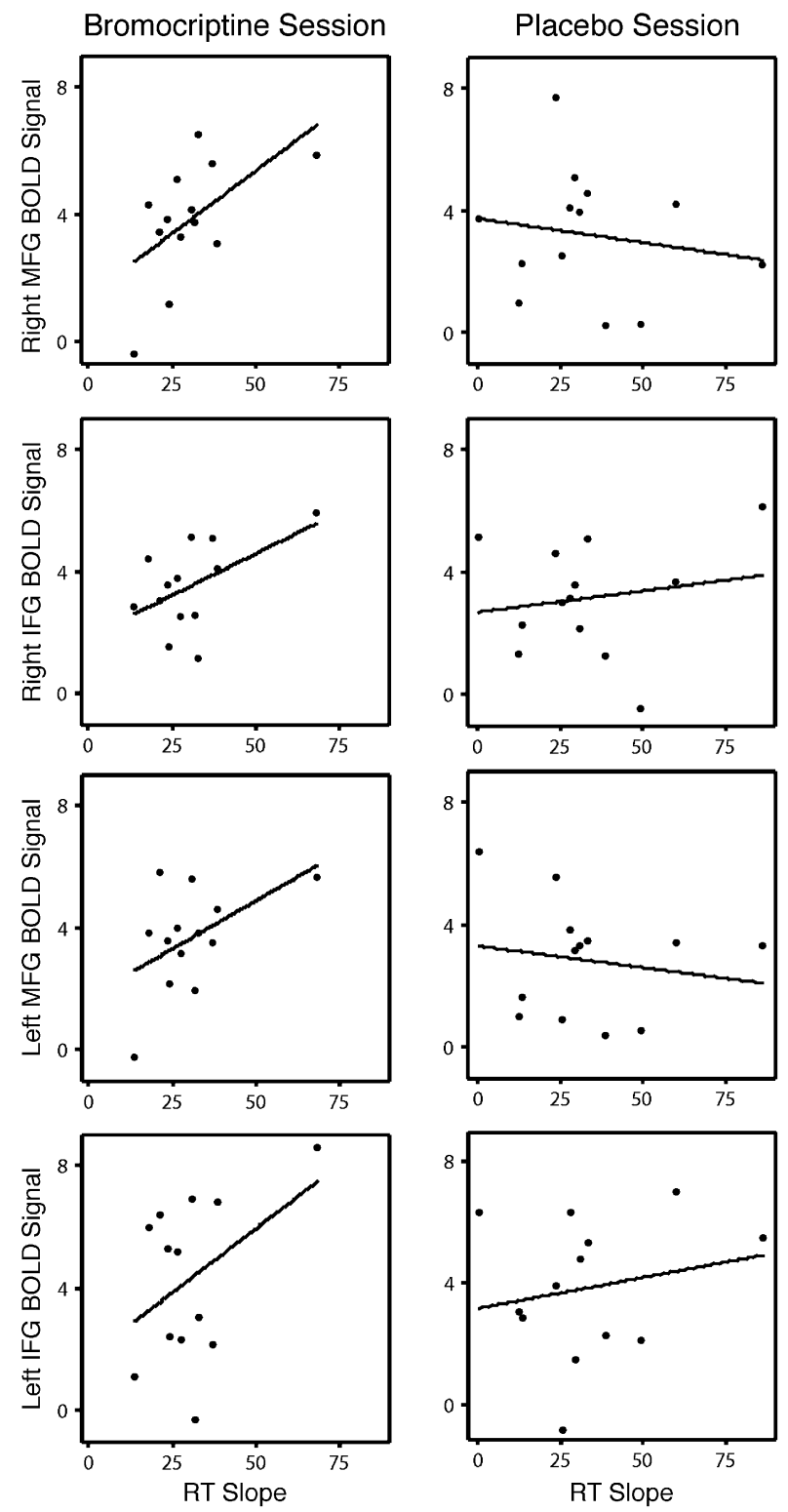

Figure 2. Correlations between RT slope and PFC activity during the bromocriptine (left panel) and placebo (right panel) sessions. With bromocriptine, slower subjects had increased and faster subjects had decreased right middle frontal gyrus (MFG) activity $(r=.56, p=.05)$. There were similar trends in other PFC regions [right inferior frontal gyrus (IFG), $r=.52, p=.07$; left MFG, $r=.50, p=.08$; left IFG, $r=.42, p=.15]$. There was no relationship between RT slope and PFC activity with placebo (all ps $>$.53).

ing span task. As was found in both our previously published study with bromocriptine (Kimberg et al., 1997) and another study using the nonspecific dopaminergic agonist methylphenidate (Mehta et al., 2000), bromocriptine improved behavioral performance for subjects with low span but tended to reduce performance for high-span subjects, especially during the most difficult higher load trials.
Although the precise relationship between working memory capacity (as indexed by span) and dopamine is unknown, we propose that individual differences in baseline PFC dopamine concentration are correlated with individual differences in working memory capacity. Support for this notion derives from studies of the individuals with polymorphisms in the catechol-O-methyltransferase (COMT) gene. COMT is the enzyme that breaks down synaptic dopamine; those individuals with the val/val polymorphism have higher COMT levels and likely have low baseline PFC dopamine concentrations, whereas those with the met/met polymorphism, and lower COMT levels, likely have a high PFC dopamine concentration. Subjects with different COMT genotypes, and likely endogenous differences in baseline PFC dopamine concentration, exhibit variations in response to a dopaminergic drug challenge similar to those observed in our study when subjects are classified by working memory span. Subjects with low PFC dopamine from the val/val polymorphism have worse performance on a working memory task than those with high PFC dopamine levels from the met/met polymorphism (Goldberg et al., 2003). Moreover, increasing dopamine levels with amphetamine improves performance for the low-dopamine val/val subjects, but impairs met/met subjects' performance in the hardest working memory task condition (Mattay et al., 2003). In the present study, we propose that low-span subjects improved on our working memory task when suboptimal PFC dopamine levels were augmented, whereas their higher span counterparts overdosed from dopaminergic augmentation, which led to performance deterioration. Our behavioral finding is also consistent with the Poewe et al. (1991) study of PD patients, which showed that dopaminergic medication improved patients' motor speeds

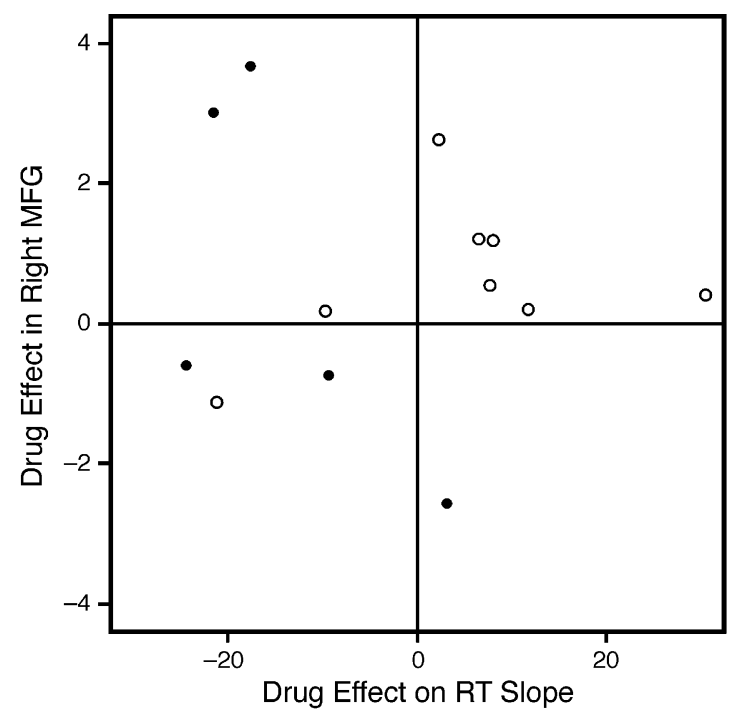

Figure 3. Plot of the effect of bromocriptine on RT slope with the effect of bromocriptine on PFC activity, showing different patterns for high-span (open dots) and low-span (shaded dots) subjects. 
but decreased their memory retrieval rates during a verbal delayed-recognition task. Thus, as has been found in animal studies that examined the effects of various dosages of dopaminergic agonist and antagonist treatments (Arnsten \& Goldman-Rakic, 1998; Sawaguchi \& GoldmanRakic, 1994; Williams \& Goldman-Rakic, 1995; Zahrt et al., 1997), each individual has an optimal dopamine concentration for optimal cognitive processing.

Our results support the hypothesis that working memory span is related to baseline dopamine availability, although they do not rule out the possibility that another mechanism independent of baseline dopamine level, such as differential sensitivities of postsynaptic dopamine receptors or altered dopamine transporter activity, also influences low-span subjects' performance. Future studies will be necessary to resolve this question, perhaps by combining working memory span measurements with COMT genotyping or PET imaging of dopamine receptor activity.

Prior studies of unmedicated subjects found differences in PFC activation patterns that were related to working memory performance. Subjects with a faster memory retrieval rate (Rypma \& D'Esposito, 1999) or with better behavioral performance (Egan et al., 2001) exhibited less PFC activity than did slower, poorer performing subjects. Here we report that the relationship of memory scanning rate at retrieval and PFC activity was influenced by dopaminergic augmentation. We found that PFC activity was correlated with memory scanning rate in the bromocriptine session (i.e., slower retrieval rate was associated with more PFC activity and faster retrieval rate with less PFC activity), whereas in the placebo session these measures were uncorrelated. We propose that this effect is related directly to the effect of bromocriptine on behavioral performance and PFC activity, depending on the subjects' span. As can be seen in Figure 3, the drug effects on retrieval rate and PFC activity in most individuals were shifted in the predicted direction, resulting in a positive correlation between these measures. Our findings may appear to conflict with those of Rypma and D'Esposito (1999), in that we did not find a significant correlation between retrieval rate and PFC activity during the placebo session. One clear difference between studies is that the previous study was performed on unmedicated patients (e.g., neither placebo nor drug). Also, since the range of RT slopes among subjects in our study (0.56-90 msec) was somewhat more restricted than in the Rypma study (9-110 $\mathrm{msec})$, perhaps we suffered from reduced power to detect such a correlation in our placebo session. It is likely that these effects were limited to the probe period, since RT slope indexes memory scanning rate, a cognitive operation most likely to be occurring as the probe stimulus is presented for comparison with the memory set. An interesting possibility for future investigation is to determine whether individual differences in neuropsychological measures like resistance to distraction may show similar relationships with activity during other task phases, such as maintenance operations occurring during the delay period.

Working memory has also been associated with PFC efficiency in studies of the COMT receptor polymorphism. Low-dopamine val/val subjects with impaired behavioral performance show increased PFC activity (Egan et al., 2001), and treatment with amphetamine that improves behavior also reduces BOLD signal, resulting in increased PFC efficiency (Mattay et al., 2000). The effect of dopamine augmentation that we observed in our study may reflect the influence of dopamine on neuronal signal enhancement. Direct application of dopamine increases task-related activity for cue-, delay-, and response-selective neurons during a delayed-response working memory task (Sawaguchi et al., 1988; Sawaguchi, Matsumura, \& Kubota, 1990a). These increases are greater for stimuli than for background activity, resulting in increased signalto-noise ratios in the PFC (Matsumura, Sawaguchi, \& Kubota, 1990; Sawaguchi, 1987).

Our finding that bromocriptine affects probe-related activity concurs with studies that have examined the possible differential functions of D1 and D2 receptor agonists. Dopamine-induced increases in cue and delay period activity are reversed by D1 antagonists but are not affected by D2 antagonists (Sawaguchi, 2001; Sawaguchi et al., 1988). In contrast, D2 agonists enhance and D2 antagonists suppress response period activity for memory-guided saccades (Wang et al., 2004). Several models of dopaminergic effects on working memory processes suggest that D1 receptor activation supports tonic activity and D2 receptors influence phasic activity (Durstewitz, Seamans, \& Sejnowski, 2000; Tanaka, 2001; West \& Grace, 2002), cellular processes that may map to working memory maintenance and updating or gating, respectively (Cohen et al., 2002; see Cools \& Robbins, 2004 , for a review). Neurophysiological studies provide support for this hypothesis, including findings that D1 receptor agonists increase activity during the delay period of working memory tasks (Sawaguchi, 2001) and that D1 stimulation alters memory fields of PFC cells with preferred response directions (Williams \& GoldmanRakic, 1995). In contrast to these delay period effects, Wang and colleagues have found no effects of D2 stimulation on mnemonic activity during the delay period. D2 receptor stimulation instead affected response period processes in monkeys. Application of a D2 agonist increased PFC saccade-related activity in a dose-dependent manner, and this increase was reversed by treatment with D2 antagonists. Our results also suggest that working memory scanning at retrieval may be influenced by phasic or D2-dependent dopamine signaling and that individual differences in scanning rates may be due to differences in D2 receptor stimulation.

Finally, since BOLD fMRI measures blood flow and not neural activity directly, and since bromocriptine may affect vascular reactivity, this potential confound is a concern in pharmacological imaging paradigms. Global 
vascular effects of bromocriptine on BOLD signal would be expected to cause consistent increases or decreases across all task periods (D'Esposito, Deouell, \& Gazzaley, 2003). Our findings are unlikely to be an artifact of changes in general vascular tone, since the drug effects that we found were limited to a particular task period. In addition, we performed direct contrasts of activity for bromocriptine and placebo collapsed across trial types and trial periods to highlight regions with global changes across each session. None of the PFC regions reported showed this type of global modulation, further strengthening the argument that these effects are not due to vascular confounds. Bromocriptine also did not affect performance on motor speed or vigilance tests, so drug effects were unlikely to be a result of changes in these basic processes.

In this event-related fMRI study, we have investigated the relationships between dopamine, PFC function, and individual differences in baseline working memory performance. Our findings from healthy young subjects given the D2 agonist bromocriptine support the role of dopamine in verbal working memory and suggest that dopamine may modulate the efficiency of retrieval of items maintained in working memory. Moreover, these data provide further evidence that individual differences in PFC dopamine receptor concentration may underlie the behavioral effects of dopaminergic stimulation on working memory function.

\section{REFERENCES}

Arnsten, A. F. T., Cai, J. X., Steere, J. C., \& Goldman-Rakic, P. S. (1995). Dopamine D2 receptor mechanisms contribute to age-related cognitive decline: The effects of quinpirole on memory and motor performance in monkeys. Journal of Neuroscience, 15, 3429-3439.

Arnsten, A. F. T., \& Goldman-Rakic, P. S. (1998). Noise stress impairs prefrontal cortical cognitive function in monkeys: Evidence for a hyperdopaminergic mechanism. Archives of General Psychiatry, $\mathbf{5 5}, 362-368$.

Brozoski, T. J., Brown, R. M., Rosvold, H. E., \& Goldman, P. S. (1979). Cognitive deficit caused by regional depletion of dopamine in prefrontal cortex of rhesus monkey. Science, 205, 929-932.

Castner, S. A., \& Goldman-Rakic, P. S. (2004). Enhancement of working memory in aged monkeys by a sensitizing regimen of dopamine D1 receptor stimulation. Journal of Neuroscience, 24, 14461450.

Cohen, J. D., Braver, T. S., \& Brown, J. W. (2002). Computational perspectives on dopamine function in prefrontal cortex. Current Opinion in Neurobiology, 12, 223-229.

Cools, R., \& Robbins, T. W. (2004). Chemistry of the adaptive mind. Philosophical Transactions of the Royal Society of London: Series A, 362, 2871-2888.

COOPER, J. R., Bloom, F. E., \& Roth, R. H. (1991). The biochemical basis of neuropharmacology (6th ed.). New York: Oxford University Press.

Curtis, C. E., \& D'Esposito, M. (2003). Persistent activity in the prefrontal cortex during working memory. Trends in Cognitive Sciences, 7, 415-423.

Daneman, M., \& Carpenter, P. A. (1980). Individual differences in working memory and reading. Journal of Verbal Learning \& Verbal Behavior, 19, 450-466.

D’Esposito, M., Deouell, L. Y., \& Gazzaley, A. (2003). Alterations in the BOLD fMRI signal with ageing and disease: A challenge for neuroimaging. Nature Reviews Neuroscience, 4, 863-872.

DruzGal, T. J., \& D'Esposito, M. (2003). Dissecting contributions of prefrontal cortex and fusiform face area to face working memory. Journal of Cognitive Neuroscience, 15, 771-784.

Durstewitz, D., \& Seamans, J. K. (2002). The computational role of dopamine D1 receptors in working memory. Neural Networks, 15, 561-572.

Durstewitz, D., Seamans, J. K., \& Sejnowski, T. J. (2000). Dopaminemediated stabilization of delay-period activity in a network model of prefrontal cortex. Journal of Neurophysiology, 83, 1733-1750.

Egan, M. F., Goldberg, T. E., Kolachana, B. S., Callicott, J. H., Mazzanti, C. M., Straub, R. E., Goldman, D., \& Weinberger, D. R. (2001). Effect of COMT Val108/158 Met genotype on frontal lobe function and risk for schizophrenia. Proceedings of the National Academy of Sciences, 98, 6917-6922.

Floresco, S. B., \& Phillips, A. G. (2001). Delay-dependent modulation of memory retrieval by infusion of a dopamine D1 agonist into the rat medial prefrontal cortex. Behavioral Neuroscience, 115, 934939.

Fuster, J. M. (1997). The prefrontal cortex: Anatomy, physiology, and neuropsychology of the frontal lobe (3rd ed.). Philadelphia: LippincottRaven.

Goldberg, T. E., Egan, M. F., Gscheidle, T., Coppola, R., Weickert, T., Kolachana, B. S., Goldman, D., \& Weinberger, D. R. (2003). Executive subprocesses in working memory: Relationship to catecholO-methyltransferase Val158Met genotype and schizophrenia. Archives of General Psychiatry, 60, 889-896.

Granon, S., Passetti, F., Thomas, K. L., Dalley, J. W., Everitt, B. J., \& RobBins, T. W. (2000). Enhanced and impaired attentional performance after infusion of D1 dopaminergic receptor agents into rat prefrontal cortex. Journal of Neuroscience, 20, 1208-1215.

HARDZINSKI, M., \& PACHELla, R. G. (1980). The manipulation of stimulus quality and the definition of stimulus encoding operations in memory scanning experiments. Perception \& Psychophysics, 27, 232-240.

IRWIN, D. E., \& PAChella, R. G. (1985). Effects of stimulus probability and visual similarity on stimulus encoding. American Journal of Psychology, 98, 85-100.

Kimberg, D. Y., D'Esposito, M., \& Farah, M. J. (1997). Effects of bromocriptine on human subjects depend on working memory capacity. NeuroReport, 8, 3581-3585.

LewIS, R., \& KupKe, T. (1977, May). The Lafayette Clinic repeatable neuropsychological test battery: Its development and research applications. Paper presented at the meeting of the Southeastern Psychological Association, Hollywood, FL.

LuCiana, M., \& Collins, P. (1997). Dopaminergic modulation of working memory for spatial but not object cues in normal humans. Journal of Cognitive Neuroscience, 9, 330-347.

Luciana, M., Depue, R. A., Arbisi, P., \& Leon, A. (1992). Facilitation of working memory in humans by a $\mathrm{D}_{2}$ dopamine receptor agonist. Journal of Cognitive Neuroscience, 4, 58-68.

Matsumura, M., Sawaguchi, T., \& Kubota, K. (1990). Modulation of neuronal activities by iontophoretically applied catecholamines and acetylcholine in the primate motor cortex during a visual reactiontime task. Neuroscience Research, 8, 138-145.

Mattay, V. S., Callicott, J. H., Bertolino, A., Heaton, I., Frank, J. A., Coppola, R., Berman, K. F., Goldberg, T. E., \& Weinberger, D. R. (2000). Effects of dextroamphetamine on cognitive performance and cortical activation. NeuroImage, 12, 268-275.

Mattay, V. S., Goldberg, T. E., Fera, F., Hariri, A. R., Tessitore, A., Egan, M. F., Kolachana, B., Callicott, J. H., \& Weinberger, D. R. (2003). Catechol O-methyltransferase val158-met genotype and individual variation in the brain response to amphetamine. Proceedings of the National Academy of Sciences, 100, 6186-6191.

McDowell, S., Whyte, J., \& D’Esposito, M. (1998). Differential effect of a dopaminergic agonist on prefrontal function in traumatic brain injury patients. Brain, 121, 1155-1164.

Mehta, M. A., Owen, A. M., Sahakian, B. J., Mavaddat, N., Pickard, J. D., \& Robbins, T. W. (2000). Methylphenidate enhances working memory by modulating discrete frontal and parietal lobe regions in the human brain. Journal of Neuroscience, 20, RC65.

Mehta, M. A., Sahakian, B. J., McKenna, P. J., \& Robbins, T. W. 
(1999). Systemic sulpiride in young adult volunteers simulates the profile of cognitive deficits in Parkinson's disease. Psychopharmacology, 146, 162-174.

Müller, U., von Cramon, D. Y., \& Pollmann, S. (1998). D1- versus D2-receptor modulation of visuospatial working memory in humans. Journal of Neuroscience, 18, 2720-2728.

Pachella, R. G., \& Fisher, D. F. (1969). Effect of stimulus degradation and similarity on the trade-off between speed and accuracy in absolute judgments. Journal of Experimental Psychology, 81, 7-9.

Phillips, A. G., Ahn, S., \& Floresco, S. B. (2004). Magnitude of dopamine release in medial prefrontal cortex predicts accuracy of memory on a delayed response task. Journal of Neuroscience, 24, 547-553.

Poewe, W., Berger, W., Benke, T., \& Schelosky, L. (1991). Highspeed memory scanning in Parkinson's disease: Adverse effects of levodopa. Annals of Neurology, 29, 670-673.

Rypma, B., \& D'Esposito, M. (1999). The roles of prefrontal brain regions in components of working memory: Effects of memory load and individual differences. Proceedings of the National Academy of Sciences, 96, 6558-6563.

SAWAGUCHI, T. (1987). Catecholamine sensitivities of neurons related to a visual reaction time task in the monkey prefrontal cortex. Journal of Neurophysiology, 58, 1100-1122.

SAWAGUCHI, T. (2001). The effects of dopamine and its antagonists on directional delay-period activity of prefrontal neurons in monkeys during an oculomotor delayed-response task. Neuroscience Research, 41, 115-128.

Sawaguchi, T., \& Goldman-RaKic, P. S. (1994). The role of D1dopamine receptor in working memory: Local injections of dopamine antagonists into the prefrontal cortex of rhesus monkeys performing an oculomotor delayed-response task. Journal of Neurophysiology, 71, 515-528.

Sawaguchi, T., Matsumura, M., \& Kubota, K. (1988). Dopamine enhances the neuronal activity of spatial short-term memory task in the primate prefrontal cortex. Neuroscience Research, 5, 465-473.

Sawaguchi, T., Matsumura, M., \& Kubota, K. (1990a). Catecholaminergic effects on neuronal activity related to a delayed response task in monkey prefrontal cortex. Journal of Neurophysiology, $\mathbf{6 3}$, 1385-1400.

Sawaguchi, T., Matsumura, M., \& Kubota, K. (1990b). Effects of dopamine antagonists on neuronal activity related to a delayed response task in monkey prefrontal cortex. Journal of Neurophysiology, 63, 1401-1412.
Sternberg, S. (1966). High-speed scanning in human memory. Science, 153, 652-654.

STERnBERG, S. (1967a). Retrieval of contextual information from memory. Psychonomic Science, 8, 55-56.

SternberG, S. (1967b). Two operations in character recognition: Some evidence from reaction-time measurements. Perception \& Psychophysics, 2, 45-53.

STERnberG, S. (1969a). The discovery of processing stages: Extensions of Donders' method. Acta Psychologica, 30, 276-315.

SternberG, S. (1969b). Memory-scanning: Mental processes revealed by reaction-time experiments. American Scientist, 57, 421-457.

TANAKA, S. (2001). Computational approaches to the architecture and operations of the prefrontal cortical circuit for working memory. Progress in Neuro-Psychopharmacology \& Biological Psychiatry, 25, 259-281.

Wang, M., Vijayraghavan, S., \& Goldman-Rakic, P. S. (2004). Selective D2 receptor actions on the functional circuitry of working memory. Science, 303, 853-856.

Watanabe, M., Kodama, T., \& Hikosaka, K. (1997). Increase of extracellular dopamine in primate prefrontal cortex during a working memory task. Journal of Neurophysiology, 78, 2795-2798

West, A. R., \& GRACE, A. A. (2002). Opposite influences of endogenous dopamine D1 and D2 receptor activation on activity states and electrophysiological properties of striatal neurons: Studies combining in vivo intracellular recordings and reverse microdialysis. Journal of Neuroscience, 22, 294-304.

Williams, G. V., \& Goldman-Rakic, P. S. (1995). Modulation of memory fields by dopamine D1 receptors in prefrontal cortex. $\mathrm{Na}$ ture, 376, 572-575.

Worsley, K. J., \& Friston, K. J. (1995). Analysis of fMRI time-series revisited-again. NeuroImage, 2, 173-181.

Zahrt, J., Taylor, J. R., Mathew, R. G., \& Arnsten, A. F. T. (1997). Supranormal stimulation of $\mathrm{D}_{1}$ dopamine receptors in the rodent prefrontal cortex impairs spatial working memory performance. Journal of Neuroscience, 17, 8528-8535.

Zarahn, E., Aguirre, G. K., \& D'Esposito, M. (1999). Temporal isolation of the neural correlates of spatial mnemonic processing with fMRI. Cognitive Brain Research, 7, 255-268.

(Manuscript received September 29, 2004; revision accepted for publication April 18, 2005.) 